\section{Sensitive scalp: An epidemio- logic study in patients with hair loss}

\author{
Anna Graziella Burroni, Ilaria Trave, \\ Astrid Herzum, Aurora Parodi \\ Section of Dermatology, Department of \\ Health Sciences (DISSAL), University of \\ Genova, IRCCS - Ospedale Policlinico \\ San Martino, Genova, Italy
}

\begin{abstract}
Sensitive scalp is a common condition defined by the presence of erythema and/or subjective symptoms as pain, pricking, burning, pruritus of the scalp elicited by triggering factors. Trichodynia is a term that describes a sensation of pain or burning of the scalp and was assumed to be part of sensitive scalp. Main goal of the study was to establish the prevalence of sensitive scalp in patients with trichological disorders. We conducted a retrospective observational study recorded: age, sex, trichological disorder (telogen effluvium, androgenetic alopecia, alopecia areata, scarring alopecia, trichotillomania) and scalp symptoms (pruritus, pain, burning and itching sensation). We studied 317 patients. 102 patients (32\%) complained of sensitive scalp. Telogen effluvium patients had a significantly higher prevalence of sensitive scalp $(\mathrm{p}<0.001)$, pain $(p=0.028)$, burning sensation $(\mathrm{p}=0.018)$, pruritus $(\mathrm{p}=0.016)$ and trichodynia $(p<0.001)$ than other patients with alopecias. Likewise, AA patients had a statistically significant higher prevalence of pruritus $(\mathrm{p}=0.0256)$ and trichodynia $(\mathrm{p}=0.0223)$ than other alopecias patients. Sensitive scalp is a frequent symptom reported by patients with hair loss. Telogen effluvium and alopecia areata seem to be most associated to sensitive scalp.
\end{abstract}

\section{Introduction}

Sensitive scalp is a common condition defined by the presence of erythema and/or subjective symptoms as pain, pricking, burning, pruritus of the scalp elicited by triggering factors as chemical factors (pollution, water, shampoos) emotional factors (stress) hormonal factors (menstrual cycle), physical factors (wind, heat, cold). Sensitive scalp is probably one of the manifestations of sensitive skin, since most of these patients declare to have sensitive skin in another body area. Predisposing factors may be either a history of atopic dermatitis or greasy/dry scalp and an associated factor may be current hair loss. ${ }^{1}$ Trichodynia is a term that describes a sensation of pain or burning of the scalp, often "spotty" or circumscribed to the areas hairs are actually coming out. ${ }^{2,3}$ Trichodynia was assumed to be part of sensitive scalp. ${ }^{1}$ Main goal of the present study was to establish the prevalence of sensitive scalp in patients with trichological disorders.

\section{Materials and methods}

We conducted a retrospective observational study on patients of the Dermatology Clinic of Genova from January 2018 to September 2019 for scarring and non-scarring alopecias. All patients gave written informed consent. For each patient, we recorded: age, sex, trichological disorder, namely telogen effluvium (TE); androgenetic alopecia (AGA); alopecia areata (AA); scarring alopecia (SA); trichotillomania (TR)). Scalp symptoms (pruritus, pain, burning and itching sensation) were also recorded. We considered patients with hair loss and pain or burning sensation of the scalp as affected by trichodynia. Patients with other cutaneous and systemic diseases and patients treated for alopecias at the time of enrollment or in the three preceding months were excluded. Hair loss was quantified by doing a pull test and hair trichoscopy during the visit and by a modified wash test (MWT). ${ }^{4}$

TE was diagnosed when patients shed $>100$ hairs at the MWT and with $<10 \%$ vellus hairs, AGA was diagnosed when they shedding $\leq 100$ hairs and with $\geq 10 \%$ vellus hairs, AGA + TE when they shed $>100$ hairs with $\geq 10 \%$ vellus hairs. ${ }^{4}$

Data were statistically analyzed using Student's t-test, Chi-squared test and, whenever needed, Fisher's exact test.

\section{Results}

We studied 317 patients (254 women, 63 men; age range 16-89 years; mean age 48 years). Eighty-three (26.2\%) had TE, 91 (28.8\%) AGA, 61 (19.2\%) TE+AGA, 49 (15.4\%) AA, 16 (5\%) SA, 17 (5.4\%) TR.

The main results are shown in Table 1. In brief, 102 patients (32\%) complained of sensitive scalp and 215 did not. No gender difference was observed $(p=0.198)$. Twenty-eight (27.45\%) were under 35 years old, $24(23.53 \%)$ from 36 to 49 years, 50 $(49.02 \%)$ over 50 years old. Of patients with sensitive scalp $39.22 \%$ had TE,
Correspondence: Astrid Herzum, Di.S.Sal Section of Dermatology, University of Genoa, San Martino Polyclinic Hospital, Largo Rosanna Benzi, 16132 Genoa, Italy.

Tel.: +39.010.5555768 - Fax: +39.0105556641 E-mail: astridherzum@yahoo.it

Key words: Sensitive scalp; telogen effluvium; hair loss.

Contributions: AGB Drafted the work and revised it critically for important intellectual content, gave final approval of the version to be published, agreed to be accountable for all aspects of the work. IT, AH, AP Drafted the work and revised it critically for important intellectual content, gave final approval of the version to be published, agreed to be accountable for all aspects of the work. All authors approved the final version of the manuscript.

Conflict of interest: The authors declare no potential conflict of interest.

Funding: None.

Ethical approval and consent to participate: Received.

Availability of data and material: Data and materials are available by the authors.

Please cite this article as: Burroni AG, Trave I, Herzum A, Parodi A. Sensitive scalp: an epidemiologic study in patients with hair loss. Dermatol Rep 2022;14:9408.

Received for publication: 21 October 2121. Accepted for publication: 5 November 2021.

This work is licensed under a Creative Commons Attribution-NonCommercial 4.0 International License (CC BY-NC 4.0).

(Copyright: the Author(s), 2022

Licensee PAGEPress, Italy

Dermatology Reports 2022; 14:9408

doi:10.4081/dr.2022.9408

Publisher's note: All claims expressed in this article are solely those of the authors and do not necessarily represent those of their affiliated organizations, or those of the publisher, the editors and the reviewers. Any product that may be evaluated in this article or claim that may be made by its manufacturer is not guaranteed or endorsed by the publisher.

$20.59 \%$ AGA, $24.49 \%$ TE+AGA, $11.76 \%$ AA, $0.99 \%$ SA, $1.96 \%$ TR.

Pruritus was complained of by 28 patients $(27.45 \%, 26$ women, 2 men) without any statistical difference $(\mathrm{p}=0.2231)$. Five $(17.86 \%)$ were under 35 years old, 5 (17.86\%) from 36 to 49 years, $18(64.28 \%)$ over 50 years old. Only patients with TE and AA had pruritus (Table 2). 
TE patients had a significantly higher prevalence of sensitive scalp $(\mathrm{p}<0.001)$, pain $\quad(p=0.028)$, burning sensation $(\mathrm{p}=0.018)$, pruritus $(\mathrm{p}=0.016)$ and trichodynia $(p<0.001)$ than other patients with alopecias. Likewise, AA patients had a statistically significant higher prevalence of pruritus $(\mathrm{p}=0.0256)$ and trichodynia $(p=0.0223)$ than other alopecias patients, but not of sensitive scalp, pain, burning $(\mathrm{p}>0.05)$.

On the contrary, in AGA patients the prevalence of sensitive scalp, pain, burning, trichodynia was not statistically higher than other alopecias patients $(\mathrm{p}>0.05)$.

Of 16 SA patients (16), only one complained of sensitive scalp. Likewise, only 2 of 17 TR patients had sensitive scalp (trichodynia).

\section{Discussion}

Sensitive scalp is a common condition in the general population (44\% of French population and $35.77 \%$ in China $)^{3,5,6}$ and its pathophysiology and etiology are still poorly understood. To our knowledge, this is the first epidemiological study of sensitive scalp in patients with hair loss. In our study, $102 / 317(32.18 \%)$ patients with hair loss had sensitive scalp, mostly women (86, $84.31 \%$ ). Why women prevail is unclear but they may have an increase in pain perception related to higher anxiety scores than men. In fact, an increased pain perception in women has been reported. ${ }^{7}$ Sensitive scalp, trichodynia, burning and itching were more prevalent in patients over 50 years $(49.02 \%$, $43.2 \%, 44.11 \%$ and $64.28 \%$, respectively). Such an increased prevalence of sensitive scalp with age may be related to the loss of nerve endings in the epidermis due to age ${ }^{8}$ and to the chronic use of irritants in shampoos. Cosmetic and care products may unbalance scalp microbiome inducing immunological or inflammatory responses, especially in elderly.,6 Moreover, elderly may have a higher perception of their own body due to a decreased level of daily activity. Among patients with sensitive scalp, trichodynia (72.52\%) (patients with pain $(39.22 \%)$ or burning $(33.33 \%)$ sensation of the scalp) is more reported than itching $(27.48 \%)$. Causes of sensory differences referred by patients with hair loss are not known but they may be related to qualitative differences in innervation. Indeed, different $\mathrm{C}$ nerve endings and their mediators bring abnormal cutaneous specific sensations. ${ }^{8}$ Most symptoms of sensitive scalp may be caused by neurogenic inflammation that probably results from the release of neurotransmitters such as substance $P$, calcitonin gene-related peptide, and vasoactive intestinal peptide inducing vasodilatation and mast cell degranulation. ${ }^{2}$

Patients with TE reported more sensitive scalp $(p=0.000007)$ than others. In addition, TE reported more pain $(\mathrm{p}=0.028585)$, burning $(\mathrm{p}=0.018361)$, itching $(\mathrm{p}=0.016089)$ and trichodynia $(p=0.0006)$ than others. In literature, the association between trichodynia and TE has

Table 1. Frequency of sensitive scalp in patients with hair loss.

\begin{tabular}{lccc} 
& Total alopecies patients & Sensitive scalp patients & Non-sensitive scalp patients \\
Total patients & 317 & $102(32.18 \%)$ & $215(67.82 \%)$ \\
Women & $254(80.13 \%)$ & $86(84.31 \%)$ & $168(78.14 \%)$ \\
\hline Men & $63(19.99 \%)$ & $16(15.69 \%)$ & $47(21.86 \%)$ \\
Mean age (years) & 48 & 43 & 53 \\
\hline Age range (years) & $16-89$ & $15-87$ & $17-89$ \\
TE & $83(26.18 \%)$ & $40(39.22 \%)$ & $43(20 \%)$ \\
AGA & $91(28.71 \%)$ & $21(20.59 \%)$ & $70(32.55 \%)$ \\
TE+AGA & $61(19.24 \%)$ & $26(24.49 \%)$ & $35(16.28 \%)$ \\
AA & $49(15.46 \%)$ & $12(11.76 \%)$ & $37(17.21 \%)$ \\
CA & $16(5.05 \%)$ & $1(0.99 \%)$ & $15(6.98 \%)$ \\
\hline TR & $17(5.36 \%)$ & $2(1.96 \%)$ & $15(6.98 \%)$
\end{tabular}

$\mathrm{TE}$, telogen effluvium; AGA, androgenetic alopecia; $\mathrm{AA}$, alopecia areata; $\mathrm{CA}$, cicatricial alopecia; $\mathrm{TR}$, trichotillomania.

Table 2. Subjective symptoms of sensitive scalp in patients with hair loss.

\begin{tabular}{lccc} 
& & Patients with trichodynia & Patients with scalp pruritus \\
Total patients & 40 & Patients with burning sensation & 28 \\
Women & $31(77.5 \%)$ & 34 & $26(92.9 \%)$ \\
\hline Men & $9(22.5 \%)$ & $29(85.3 \%)$ & $2(7.1 \%)$ \\
Mean age (years) & 43 & $5(14.7 \%)$ & 59 \\
\hline Age range (years) & $17-81$ & 52 & $21-89$ \\
Isolated TE & $15(37.5 \%)$ & $18-88$ & $10(35.7 \%)$ \\
\hline Isolated AGA & $10(25 \%)$ & $11(32.4 \%)$ & $0(0 \%)$ \\
TE+AGA & $10(25 \%)$ & $5(14.7 \%)$ & $11(39.3 \%)$ \\
AA & $3(7.5 \%)$ & $2(5.9 \%)$ & $7(25 \%)$ \\
SA & $0(0 \%)$ & $1(2.9 \%)$ & $0(0 \%)$ \\
TR & $2(5 \%)$ & $0(0 \%)$ & $0(0 \%)$
\end{tabular}

$\mathrm{TE}$, telogen effluvium; $\mathrm{AGA}$, androgenetic alopecia; $\mathrm{AA}$, alopecia areata; $\mathrm{CA}$, cicatricial alopecia; $\mathrm{TR}$, trichotillomania. 
already been reported, ${ }^{3,8,9,10}$ although trichodynia may be also signaled in AA, ${ }^{11}$ AGA and AGA+TE. ${ }^{9,12}$ Researchers reporting trichodynia in AGA, however, did not consider the possibility of the TE+AGA association. The difficulties about studying trichodynia are due to its uncertain pathogenesis. Ericson et al. demonstrated that patients with trichodynia have an increase of a neuropeptide release (stress-related Neuropeptide (SP)) by cutaneous peripheral nerve terminals that induces and perpetuates an inflammation of hair follicles inducing abnormalities in the hair cycle. ${ }^{3,13}$ This hypothesis may explain the concurrence of trichodynia and inflammatory alopecia such as AA ( $\mathrm{p}=0.0223)$, as in our case. Hoss and Segal considered trichodynia associated to an underlying psychiatric disorder such as depression (TE group), generalized anxiety, somatoform disorder and obsessive personality disorder (AGA group). ${ }^{10,14}$ We agree with the hypothesis of a multifactorial pathogenesis.

As for AGA patients, they report more frequently itching $(p=0.0009)$ due to a concomitant seborrhea and dandruff and to the use of irritant shampoos to obtain itching relief. By contrast, AA patients report itching $(p=0.0256)$ which often precedes the development of new patches. The itching in AA patients may be caused by mast cell release of histamine and tryptase as well as lymphocytic infiltration with release of IL$31 .{ }^{15}$ Generalized itching preceding AA universalis has been reported even in the XVI century.

\section{Conclusions}

Sensitive scalp is a frequent symptom reported by patients with hair loss. The pruritus seems to prevail in patients with AGA, while trichodynia prevails in disorders in which inflammation is obvious or highly suspected. A prospective study aimed to establish possible associations between the intensity of symptoms and hair loss is in program.

\section{References}

1. Misery L, Sibaud V, Ambronati M, et al. Sensitive scalp: does this condition exist? An epidemiological study. Contact Dermatitis 2008;58:234-8.

2. Sulzberger MB, Witten VH, Kopf AW. Diffuse alopecia in women. Its unexplained apparent increase in incidence. Arch Dermatol 1960;81:556-60.

3. Rebora A, Semino MT, Guarrera M. Trichodynia. Dermatology 1996;192: 292-3.

4. Rebora A. Telogen effluvium: a comprehensive review. Clin Cosmet Investig Dermatol 2019 21;12:583-90.

5. Guichard A, Ma L, Tan Y, et al. What if scalp flora was involved in sensitive scalp onset? Int J Cosmet Sci. 2016;38: 429-30.

6. Ma L, Guichard A, Cheng Y, et al. Sensitive scalp is associated with excessive sebum and perturbed microbiome. J Cosmet Dermatol 2019;18:922-8.
7. Willimann B, Trüeb RM. Hair pain (trichodynia): frequency and relationship to hair loss and patient gender. Dermatology. 2002;205:374-7.

8. Misery L, Rahhali N, Ambonati M, et al. Evaluation of sensitive scalp severity and symptomatology by using a new score. J Eur Acad Dermatol Venereol 2011;25:1295-8.

9. Baldari M, Montinari M, Guarrera M, Rebora A. Trichodynia is a distinguishing symptom of telogen effluvium. J Eur Acad Dermatol Venereol 2009;23:733-4.

10. Kivanç-Altunay I, Savaş C, Gökdemir $\mathrm{G}$, et al. The presence of trichodynia in patients with telogen effluvium and androgenetic alopecia. Int $\mathrm{J}$ Dermatol 2003;42:691-3.

11. Bolduc C, Lui H, Shapiro J. Alopecia Areata. E-Medicine from WebMD, 2006.

12. Defrin R, Lurie R. Indications for peripheral and central sensitization in patients with chronic scalp pain (trichodynia). Clin J Pain 2013;29:417-24.

13. Ericson M, Gabrielson A, Worel S, et al. Substance P (SP) in innervated and noninnervated blood vessels in the skin of patients with symptomatic scalp. Exp Dermatol 1999;8:344-5.

14. Hoss D, Segal S. Scalp dysesthesia. Arch Dermatol. 1998;134:327-30.

15. Vázquez-Herrera NE, Sharma D, Aleid NM, Tosti A. Scalp Itch: A Systematic Review. Skin Appendage Disord. 2018;4:187-99. 